\author{
Christian A. Schmittinger \\ Christian Torgersen \\ Günter Luckner \\ Daniel C. H. Schröder \\ Ingo Lorenz \\ Martin W. Dünser
}

\section{Response to Salvadori et al.: adverse cardiac events during catecholamine therapy: a role for hydrocortisone?}

Accepted: 1 July 2012

Published online: 7 August 2012

(C) Copyright jointly held by Springer and ESICM 2012

This reply refers to the comment available at: doi:10.1007/s00134-012-2663-4.

Dear Editor,

We thank Dr. Salvadori and his colleagues for their interest in our work [1] and the constructive comments. In view of the evolving controversy regarding the effects of hydrocortisone therapy in sepsis during the study period (January to December 2009), the use of hydrocortisone was strictly reserved for patients with septic shock requiring escalating norepinephrine doses. This explains why only one patient suffering from septic shock received hydrocortisone (continuous infusion of $200 \mathrm{mg} /$ day) at study enrollment. Unfortunately, this precludes evaluation of the influence of hydrocortisone on the binary occurrence of adverse cardiac events in our study population. At the occurrence of an adverse cardiac event, 15 of 21 septic shock patients $(71.4 \%)$ received a continuous hydrocortisone infusion at a median (interquartile range, IQR) daily dose of 192 (192-288) $\mathrm{mg}$. The absolute number of adverse cardiac events did not differ between septic shock patients with or without hydrocortisone therapy [4 (2.5-6) versus 3 $(2-7)]$ in both an unadjusted $(p=0.64)$ and adjusted $(p=0.12)$ model (model adjustment for Simplified Acute Physiology Score II, need for renal replacement therapy, presence of chronic liver diseases). Interestingly, however, patients on hydrocortisone developed adverse cardiac events at higher median vasopressor load $[0.48$

$(0.36-0.54) \mu \mathrm{g} / \mathrm{kg} / \mathrm{min}]$ than patients who did not receive hydrocortisone [0.18 $(0.06-0.43) \mu \mathrm{g} / \mathrm{kg} / \mathrm{min}]$ in both an unadjusted $(p=0.005)$ and adjusted model $(p=0.006)$. The type of adverse cardiac event had no influence on this association ( $p=0.17$ and $p=0.13$, respectively). Carrying the hypothesis of Salvadori and colleagues on a potentially protective effect of

hydrocortisone on the occurrence of adverse cardiac events during catecholamine vasopressor therapy further, one may speculate that hydrocortisone raised the individual threshold at which catecholamine vasopressors may induce adverse cardiac events in our study patients with septic shock. Taking the post hoc character of this analysis and the low number of septic shock patients developing adverse cardiac events $(n=21)$ into consideration, these data must be regarded as purely hypothesis-generating. Further studies are, therefore, needed to evaluate the influence of a hydrocortisone therapy on the occurrence of adverse (cardiac) events during catecholamine vasopressor therapy in septic shock.

\section{Reference}

1. Schmittinger CA, Torgersen C, Luckner G, Schröder DCH, Lorenz I, Dünser MW (2012) Adverse cardiac events during catecholamine vasopressor therapy: a prospective observational study. Intensive Care Med 38:950-958. doi:10.1007/s00134-012-2531-2

\section{A. Schmittinger - C. Torgersen} Department of Intensive Care Medicine, Inselspital, Bern University Hospital and University of Bern, Bern, Switzerland

\section{A. Schmittinger (®)}

Department of Anaesthesiology, Surgical Intensive Care Medicine, and Rescue Medicine, Lucerne Cantonal Hospital, Lucerne, Switzerland e-mail: christian.schmittinger@luks.ch Tel.: +41-41-2054901

C. Torgersen - G. Luckner .

D. C. H. Schröder · I. Lorenz

Department of Anaesthesiology and Critical Care Medicine, Innsbruck Medical

University, Innsbruck, Austria

\section{W. Dünser}

Department of Anaesthesiology, Perioperative and Intensive Care Medicine, Salzburg General Hospital and Paracelsus Private Medical University,

Salzburg, Austria 